\title{
Valutazione dell'impatto da odori negli impianti di trattamento dei rifiuti solidi urbani
}

\author{
Massimo Noschese ${ }^{1}$ \\ ${ }^{1}$ University of Salerno
}

\begin{abstract}
La valutazione delle emissioni odorigene e dei relativi impatti viene effettuata utilizzando i fattori di emissioni di odori (OEF), cosi come riportato in numerosi studi scientifici, che tengono conto solo della capacità dell'impianto per la quantificazione dell'indice di attività.

Le attività di ricerca, presenti in questo studio, sono state improntate all'individuazione delle principali fonti di odori e le loro relative concentrazioni ed emissioni provenienti da un grande Impianto di Trattamento Integrato Anaerobico-Aerobico. Tali fonti di odori sono state valutate attraverso l'ofattometria dinamica.

Gli OEF rilevanti sono stati stimati utilizzando un set di dati sperimentali e confrontati con quelli teorici proposti negli studi di letteratura su impianti di trattamento simili.

I risultati hanno mostrato come le principali emissioni di odori sono associate al processo di compostaggio.
\end{abstract}

\section{Introduzione}

All'interno del sistema integrato di gestione dei rifiuti da raccolta differenziata si adottano misure ed azioni intese ad ottenere un recupero di materia e di energia. Questi trattamenti vengono effettuati in impianti che combinano trattamenti anaerobico (digestione) ${ }^{1}$ con recupero di energia e trattamenti aerobici (compostaggio) ${ }^{2}$ con recupero di materia. Secondo i dati del ISPRA (Istituto superiore per la protezione e la ricerca ambientale) in Italia questi impianti integrati sono aumentati in modo significativo negli ultimi anni.

I potenziali impatti ambientali di questi impianti integrati anaerobiciaerobici (AATP) sono generalmente gli odori ${ }^{3},{ }^{4}$. Gli odori sono un disturbo per la popolazione che abita nelle vicinanze della struttura. 


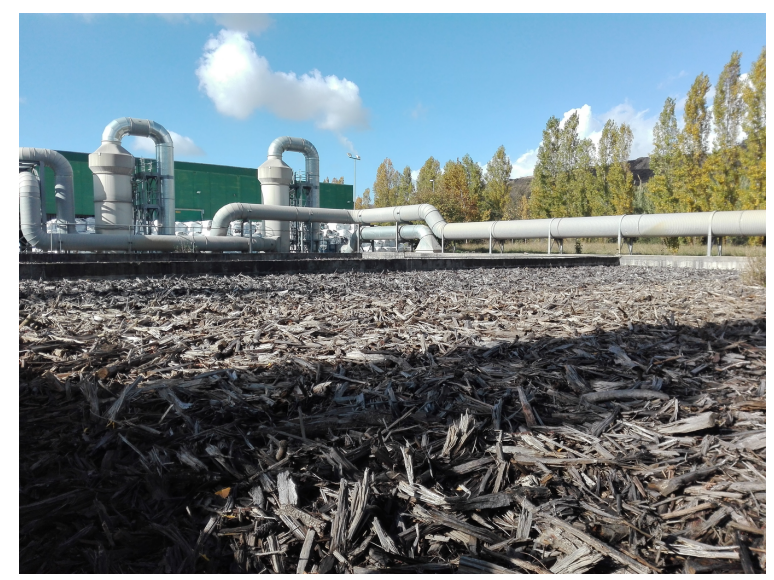

Figure 1: Impianto di trattamento aree esauste

Per limitare le problematiche relative alla diffusione degli odori ne vengono valutate le emissioni ${ }^{5},{ }^{6}$.

Nella letteratura scientifica attuale si osserva una scarsità di dati relativi alla caratterizzazione di emissioni di odore da impianti integrati anaerobiciaerobici (AATPs). Alcuni studi scientifici ${ }^{7}$ propongono l'utilizzo di Fattori di Emissione dell'Odore (OEFs) come strumento atto a definire le strategie più appropriate per il controllo dell'emissione negli impianti esistenti o per la previsione dell'impatto degli odori in impianti nuovi ${ }^{8},{ }^{9}$. Gli OEF sono definiti come il rapporto tra tasso di emissione degli odori (OER) e un indice di attività rappresentativo dell'impianto ${ }^{9}$. Gli studi attuali indicano la capacità dell'impianto come possibile indice di attività $8,9,10, \mathrm{i}$ quali sono spesso sostituiti, per limitare l'errore, con dati ottenuti sperimentalmente ${ }^{11}$. Le principali fonti di odore con le relative concentrazioni sono state analizzate attraverso l'olfattometria dinamica. Queste misurazioni sono state studiate e confrontate con quelle teoriche proposte nella letteratura attuale, utilizzando la capacità complessiva dell'impianto come indice di attività. Obiettivo di questo studio è contribuire alla conoscenza e alla comprensione delle emissioni di odori di un impianto integrato di trattamento dei rifiuti, fornendo dati che possono essere adottati per la previsione dell'indice di attività dell'impianto stesso e utilizzati per la valutazione dell'impatto olfattivo. 


\section{Programma di monitoraggio e valutazione}

La potenzialità dell'impianto è pari a circa $30.000 \mathrm{t} / \mathrm{a}$, di cui all'incirca l' $80 \%$ è la frazione organica dei rifiuti solidi (OFSW) e il rimanente e scarto verde. L'impianto è strutturato con tre linee di trattamento, una dedicata al processo aerobico, uno per il trattamento anaerobico e una fase comune per entrambi i processi ovvero il trattamento di aree esauste con l'ausilio di scrubber e biofiltri.

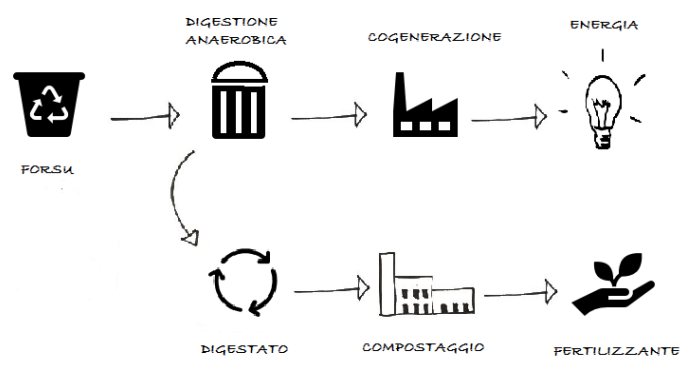

Figure 2: Linea di trattamento

Il monitoraggio ha avuto una durata di un anno con una frequenza di campionamento mensile. Sono state analizzate le unità di trattamento con il maggior tasso di emissioni di odori: punti di stoccaggio (rifiuti organici, materiale strutturante, prodotto finale compost) e biofiltri.

Contemporaneamente al campionamento degli odori è stata analizzata anche la frazione organica solida, valutandone il contenuto di materia organica. I campioni raccolti sono 72 di odori e 12 di OFSW.

Le unità esaminate, sono state campionate utilizzando una camera di flusso SF450 (Scentroid, CDN), con una pompa a vuoto avente un sacchetto di 10 L. I parametri operativi della camera di flusso utilizzata sono la superficie coperta pari a $0,155 \mathrm{~m}^{2}$ e un flusso d'aria neutro all'ingresso pari a 3,9 Ipm.

Le analisi della composizione dell'OFSW sono state fatte su un campione iniziale di $300 \mathrm{~kg}$ secondo il metodo descritto nell'allegato B del DGRV $568 / 2005$ adottata dal Consorzio dei compostatori italiani (CIC). 
In questo studio ${ }^{12}$ le concentrazioni degli odori sono state valutate mediante analisi olfattometriche dinamiche secondo la norma EN 13725:2003. È stato utilizzato l'olfattometro TO8 (ECOMA, D), basato sul metodo "si/no"; con I 'ausilio di un gruppo di valutazione composto da quattro persone addestrate. Tutte le misurazioni sono state analizzate entro 14 ore dal campionamento.

Il calcolo delle emissioni di odori (OER, ou $\left.\mathrm{s}^{-1}\right)$ del sistema studiato è il prodotto tra il tasso di emissione specifico dell'odore (SOER, ou $\mathrm{s}^{-1} \mathrm{~m}^{-2}$ ) e l'area della superficie di emissione $\left(A_{E}, \mathrm{~m}^{2}\right)$ che si è campionata. SOER è stato calcolato moltiplicando la concentrazione olfattiva, misurata all'uscita della camera di flusso, (COD, ou $\mathrm{m}^{-3}$ ) per la portata dell'aria aspirata $\left(\mathrm{m}^{3}\right)$, il tutto diviso l'area di base del corpo della camera $\left(\mathrm{m}^{2}\right)$.

Le $\mathrm{OEF}_{\mathrm{S}}$ sono state calcolate dividendo il tasso di emissione di odori $\left(\mathrm{OER}, \mathrm{ou}_{\mathrm{E}} \mathrm{s}^{-1}\right)$ per un indice specifico di attività $(A)$, che è rappresentativo dell'impianto esaminato e associato a una quantità di odore emesso. In seguito, sono stati valutati anche gli OEF per ciascuna fonte oggetto del monitoraggio dividendo il valore medio OER, determinato per ciascuna fonte durante l'intero periodo di monitoraggio, per la capacità dell'impianto.

Per migliorare e ridurre il margine di errore nella determinazione degli OEF è stato calcolato il SOEF adottando come indice uno specifico parametro rappresentativo della materia organica (OM) presente nell'OFSW. L'OM è la percentuale della quantità totale di OFSW presente durante il campionamento degli odori ed espressa in tonnellate. La validità del nuovo parametro proposto come indice di attività è stata valutata attraverso studi di correlazione, analizzando la sua dipendenza dall'emissione di odori calcolata. I coefficienti di correlazione lineare $\left(R^{2}\right)$, con un valore superiore a 0,95 sono stati considerati indicatori di buona dipendenza.

I risultati ottenuti mostrano che la concentrazione massima di odori tra le fonti esaminate è l'unità di ricezione dei rifiuti OFSW (2545 ouE m³), mentre la più bassa è quella misurata al biofiltro dove viene trattata l'aria esausta del secondo capannone di stagionatura $\left(67 \mathrm{ouE} \mathrm{m}^{-3}\right)$. La maggiore variabilità delle concentrazioni di odori è alla ricezione dei rifiuti verdi. Le concentrazioni odorigene misurate ai biofiltri non hanno mai superato il valore di $300 \mathrm{ouE} \mathrm{\textrm {m } ^ { - 3 }}$, fissato come limite dalle attuali linee guida in Italia.

I dati ottenuti mostrano valori diversi da quelli presentati nella letteratura attuale per un trattamento di impianti simili ${ }^{9}$ considerando la stessa capacità dell'impianto. La differenza tra i valori attuali e quelli teorici è probabilmente anche relativa alla diversa strumentazione di campionamento utilizzata in questo studio da quella $\mathrm{di}^{9}$ (camera di flusso vs. galleria del vento), che può portare a valori OER diversi, come dimostrato dallo studio di ${ }^{13}$.

I risultati indicano per i dati ottenuti e il tipo di OFSW esaminato l'esistenza di una proporzionalità tra l'OER calcolato e I'OM determinato con coeffi- 
cienti di correlazione lineari $\left(R^{2}\right)$ pari allo 0,967 e di conseguenza la materia organica può pertanto essere considerata come un indice di attività $(A)$ per la fase di ricevimento dell'OFSW per prevedere le emissioni odorigene (OER).

\section{Conclusioni}

Sono stati determinati e calcolati per un grande AATP di rifiuti solidi organici le concentrazioni e le emissioni di odore delle unità di trattamento con una maggiore tendenza alle emissioni.

I risultati ottenuti mostrano che le principali fonti di odore sono le unita di raccolta rifiuti (OFSW e rifiuti verdi). Ė stato valutato l'OEF attraverso un set di dati sperimentali e sono stati confrontati con i dati teorici che vengono proposti nella letteratura corrente sugli impianti MBT, utilizzando come indice di attività la capacità dell'impianto. II confronto degli OEF valutati con quelli teorici hanno mostrato valori molto diversi. Lo studio conferma l'accuratezza dell'uso degli OEF dipendente dalla possibilità di questo parametro di rappresentare completamente le emissioni di odori. L'Utilizzo della capacità dell'impianto come indice di attività per calcolare solo I'OEF è molto riduttivo in quanto può essere applicato a tutte le fonti di un impianto per prevedere le loro emissioni di odori. Si suggerisce quindi di utilizzarlo per identificare le SOEFs.

Da questo studio si evince l'esistenza di una relazione di proporzionalità tra I'OER calcolato e l'OM determinato con coefficienti di correlazione lineari $\left(R^{2}\right)$ pari a 0,967 e di conseguenza la possibilità di utilizzare questo nuovo parametro $(\mathrm{OM})$ come indice di attività per calcolare l'OEF e l'OER per questa unità. Per ratificare ciò che è stato definito in forma sperimentale in questo lavoro sono necessari studi aggiuntivi su impianti simili.

\section{References}

1.A.Cesaro \& V.Belgiorno. Pretreatment methods to improve anaerobic biodegradability of organic municipal solid waste fractions. Chemical Engineering Journal 240, 24-37 (2014).

2.A.Cesaro, A.Conte, V.Belgiorno, A.Siciliano \& M.Guida. The evolution of compost stability and maturity during the full-scale treatment of the organic fraction of municipal solid waste. Journal of Environmental Management 232, 264-270 (2019).

3.W.Bidlingmaier. Odour emissions from composting plants. in The Science of Composting and Springer Netherlands (1996).

4.V.Belgiorno, V.Naddeo \& T.Zarra. Odour Impact Assessment Handbook. Wiley \& Son (London. ISBN: 978-1-119-96928-0, 2012). 
5.C.A.Rincòn et al.. Chemical and odor characterization of gas emissions released during composting of solid wastes and digestates. Journal of Environmental Management 233, 39-53 (2019).

6.T.Zarra, Naddeo, V., V.Belgiorno, M.Reiser \& M.Kranert. Odour monitoring of small wastewater treatment plant located in sensitive environment. Water Science and Technology 58, (2008).

7.T.Zarra, V.Naddeo, G.Oliva \& V.Belgiorno. Odour Emissions Characterization for Impact Prediction in Anaerobic-Aerobic Integrated Treatment Plants of Municipal Solid Waste. Chemical Engineerin 54, (2016).

8.L.Capelli, S.Sironi \& Rosso, R. D. Odour Emission Factors: Fundamental Tools for Air Quality Management. in Chemical Engineering Transactions (http://doi.org/10.3303/CET1440033, 2014).

9.S.Sironi, L.Capelli, Centola, P., Rosso, R. D. \& Grande, M. I. Odour emission factors for the prediction of odour emissions from plants for the mechanical and biological treatment of MSW. Atmospheric Environment 40, 7632-7643 (2006)

10.E.Epstein. The Science of Composting. in Technomic Publishing Company (Inc., Lancaster, PA, USA, 1997).

11.S.Sironi, L.Capelli, Cèntola, P., Rosso, R. D. \& Grande, M. I. Odour emission factors for assessment and prediction of Italian rendering plants odour impact. Chemical Engineering Journal 131, 225-231 (2007).

12.T.Zarra, M.Reiser, V.Naddeo, V.Belgiorno \& M.Kranert. A comparative and critical evaluation of different sampling materials in the measurement of odour concentration by dynamic olfactometry. Chemical Engineering Transactions 30, (2012).

13.N.Hudson et al.. Comparison of odour emission rates measured from various sources using two sampling devices. Bioresource Technology 100, 118-124 (2009). 